\title{
HDM4 is overexpressed in mantle cell lymphoma and its inhibition induces p21 expression and apoptosis
}

Mei Liang ${ }^{1}$, Xin $\mathrm{Han}^{2}$, Saroj Vadhan-Raj ${ }^{3}$, Martin Nguyen ${ }^{1}$, Yu H Zhang ${ }^{1}$, Michael Fernandez ${ }^{1}$, Elias Drakos ${ }^{1}$, Sergej N Konoplev ${ }^{1}$, C Cameron Yin ${ }^{1}$, Roberto N Miranda ${ }^{1}$, Timothy J McDonnell ${ }^{1}$, L Jeffrey Medeiros ${ }^{1}$ and Carlos E Bueso-Ramos ${ }^{1}$

${ }^{1}$ Department of Hematopathology, The University of Texas MD Anderson Cancer Center, Houston, TX, USA; ${ }^{2}$ Department of Laboratory Medicine, The University of Texas MD Anderson Cancer Center, Houston, TX, USA and ${ }^{3}$ Department of Cytokine and Supportive Oncology, The University of Texas MD Anderson Cancer Center, Houston, TX, USA

In mouse models and cell lines, murine double minute 2 (MDM2) and MDM4 have been shown to synergistically promote proteasome-mediated degradation of p21 and p53. MDM4 also inhibits p53-mediated transcriptional activation of p21. p53 expression results in increased p21 expression, a negative cell-cycle regulatory protein and an inhibitor of cyclin D1. As mantle cell lymphoma is characterized by cyclin D1 overexpression, we assessed for human homolog of MDM4 (HDM4) expression and its effect on p21 in mantle cell lymphoma. Using immunohistochemical methods, in reactive lymph nodes $(n=19)$ germinal center cells strongly expressed HDM4 in the nucleus and the cytoplasm, but mantle zone B-cells were only dimly positive. In mantle cell lymphoma tumors, aberrant HDM4 nuclear expression was observed in 18 of 19 (95\%) cases. In contrast, HDM4 in other B-cell non-Hodgkin lymphoma types retained its normal pattern of expression. To further characterize the differential upregulation of HDM4 in mantle cell lymphoma, HDM4 was assessed by quantitative real-time polymerase chain reaction in four mantle cell lymphoma cell lines (Granta 519, Z-138, SP-53, and Mino) and six mantle cell lymphoma tumors. Both the splicing variant HDM4-S, containing only the p53-binding domain, and full length HDM4 were increased compared with normal CD19+ B-cells $(P<0.05)$. Using small interfering RNA to inhibit HDM4 in the SP53 and Mino cell lines showed increased p21 and active caspase-3, the latter indicating increased apoptosis. Our results show that HDM4 is overexpressed in mantle cell lymphoma and, at least in part, exerts its effect by suppressing p21 expression, thereby enhancing cell-cycle progression. Inhibition of HDM4 may serve as a potential approach in the design of therapy for patients with mantle cell lymphoma. Modern Pathology (2010) 23, 381-391; doi:10.1038/modpathol.2009.170; published online 8 January 2010

Keywords: mantle cell lymphoma; HDM4; HDMX; HDM2; p53; p21

Mutational inactivation of p53 is only one mechanism of disrupting p53 function in tumorigenesis. ${ }^{1}$ An alternative mechanism is overexpression of negative regulators of $\mathrm{p} 53$, such as human homolog of murine double minute 4 (HDM4, also known as HDMX) and HDM2 (human homolog of MDM2). The

Correspondence: Dr CE Bueso-Ramos, MD, PhD and X Han, MD, Departments of Hematopathology and Laboratory Medicine, Units 72 and 37, respectively, The University of Texas MD Anderson Cancer Center, 1515 Holcombe Boulevard, Houston, TX 77030, USA.

E-mail: cbuesora@mdanderson.org

Received 20 April 2009; revised 8 July 2009; accepted 6 August 2009; published online 8 January 2010
HDM4 gene is located at chromosome $1 \mathrm{q} 32^{2,3}$ and this locus is a target of amplification in malignant gliomas that have no p53 mutations or MDM2 amplification. ${ }^{4}$ Furthermore, the HDM4 gene has been shown to be amplified in breast carcinoma ${ }^{5}$ and soft tissue sarcoma, ${ }^{6}$ and HDM4 protein is aberrantly expressed in several human tumor cell lines. ${ }^{7,8}$ These data suggest that the HDM4 gene is dysregulated in human neoplasms and may contribute to tumor development or progression.

HDM4 inhibits p53-mediated transcriptional activities, such as cell-cycle arrest and apoptosis, ${ }^{9-12}$ and HDM2 predominantly affects the stability of p53 by degradation through its E3 ubiquitin ligase 
activity. ${ }^{13}$ Knockout of either murine double minute 4 (MDM4) or MDM2 in mice (MDM4-/- or MDM2-/-) results in embryonic lethality, which can be reversed by deletion of p53. ${ }^{14}$ It is also known that MDM4 stimulates MDM2-mediated ubiquitination of p53, and is involved in the degradation of both p53 and MDM2, suggesting that MDM4 is an important regulator of the p53-MDM2 pathway. ${ }^{15}$ Recent data show that MDM4 and MDM2 independently and cooperatively regulate proteasome-mediated degradation of the cyclin-dependent kinase inhibitor, p21, in the G1 and early S phases of the cell cycle. ${ }^{16}$ p21 physiologically inhibits cyclin D1.

Mantle cell lymphoma is characterized by the $t(11 ; 14)(q 13 ; q 32)$ that juxtaposes the CCND1 gene at chromosome 11q13 with the immunoglobulin heavy-chain gene at $14 q 32$, resulting in overexpression of cyclin D1. This genetic event is present in virtually all cases of mantle cell lymphoma, but appears insufficient for lymphomagenesis in transgenic mouse experiments. ${ }^{17,18}$ Additional cytogenetic $^{19}$ and molecular alterations occur in mantle cell lymphoma, and many of these alterations disrupt cell-cycle regulation or interfere with the cellular response to DNA damage. ${ }^{20}$ p53 is a key transcription factor that is upregulated in response to cellular stress such as DNA damage leading to cell-cycle arrest or apoptosis. Although p53 inactivation is rarely observed in typical mantle cell lymphoma cases with low proliferative activity, p53 mutations or overexpression occur in up to $30 \%$ of blastoid mantle cell lymphoma cases that have a high proliferation rate. ${ }^{21,22}$ Recent gene expression studies of mantle cell lymphoma have shown that the HDM2 expression level is an independent prognostic factor. ${ }^{23}$ As an alternative mechanism to p53 inactivation, high levels of HDM2 and HDM4 may be important in the pathogenesis of mantle cell lymphoma.

Our previous report showed that HDM4 is widely expressed in adult B-lymphoblastic leukemia/lymphoma. ${ }^{24}$ However, the expression pattern of HDM4 in various types of non-Hodgkin lymphoma is unknown. The primary goal of this work is to characterize HDM4 expression and explore its potential role in the pathogenesis of mantle cell lymphoma, a lymphoma with known poor prognosis for which novel therapeutic strategies are needed.

\section{Materials and methods}

We studied four mantle cell lymphoma cell lines, Mino, SP-53, Granta 519, and Z-138, cultured as described earlier. ${ }^{25,26}$ We also assessed 25 mantle cell lymphoma tumors. These cases included formalin-fixed and routinely processed tissue blocks of 19 tumors and 6 peripheral blood samples obtained from patients with leukemic phase of mantle cell lymphoma. For comparison, we examined 19 reactive lymph nodes and 53 cases of other B-cell
Table 1 Summary of HDM4 expression in reactive lymph nodes and B-cell non-Hodgkin lymphomas

\begin{tabular}{lc}
\hline Subtype & Positive/total case \\
\hline Mantle cell lymphoma* & $18 / 19(95)$ \\
Reactive & \\
$\quad$ Germinal center & $19 / 19(100)$ \\
Mantle zone* & $6 / 19(32)$ \\
Marginal zone & $19 / 19(100)$ \\
Small lymphocytic lymphoma/CLL & $8 / 11(72)$ \\
Follicular lymphoma & $13 / 16(81)$ \\
Grade 1 & $6 / 6(100)$ \\
Grade 2 & $6 / 7(86)$ \\
Grade 3 & $1 / 3(33)$ \\
Marginal zone B-cell lymphoma & $5 / 6(83)$ \\
Diffuse large B-cell lymphoma & $16 / 20(80)$
\end{tabular}

${ }^{*} P<0.001$.

lymphoma types (Table 1). All tumor specimens were obtained from patients before therapy at The University of Texas MD Anderson Cancer Center from 2001 to 2007. The diagnosis of mantle cell lymphoma and other B-cell non-Hodgkin lymphoma types was based on a combination of clinical, morphologic, and immunophenotypic criteria as defined by the 2008 World Health Organization Classification. All cases of mantle cell lymphoma carried the $t(11 ; 14)(q 13 ; q 32)$ and overexpressed cyclin D1. The study was approved by the Institutional Review Board.

\section{Immunohistochemical Analysis}

Whole tissue sections rather than tissue microarray sections were studied to better appreciate the expression pattern of HDM4 in different lymph node compartments. Immunostaining was performed using $5 \mu \mathrm{m}$ thick, formalin-fixed, paraffinembedded tissue sections, epitope retrieval with Diva decloacker buffer and a decloacking chamber (Biocare Medical, Concord, CA, USA), and the Mach 3 system (Biocare Medical). Staining was performed using the Autostainer Plus (DakoCytomation, Carpinteria, CA, USA). The washing buffer used was $0.05-\mathrm{M}$ Tris-buffered saline supplemented with $0.05 \%$ Tween; 3,3'-diaminobenzidine tetrahydrochloride was used as the chromogen (Liquid $\mathrm{DAB}+$ Subtrate Chromogen System, DakoCytomation) and all tissue sections were counterstained with hematoxylin.

Single antibody staining of tissue sections The antibodies used were specific for HDM4 (1:1500, rabbit anti-Hdmx/MDM4, Bethyl Laboratories, Montgomery, TX, USA), the splicing variant (HDM4-S, 1:100, generated by Dr X Han), and p21 (1:50, DakoCytomation). Cases were considered positive for HDM4 if $25 \%$ or more of the tumor 
cells were positive. Both nuclear and cytoplasmic staining was recorded separately. Cases were considered positive for p21 if 5\% or more of the tumor cells were positive.

Double antibody staining of tissue sections The first antibody, HDM4, was applied as described above. After the reaction was visualized with diaminobenzidine tetrahydrochloride (Liquid DAB + Subtrate Chromogen System, DakoCytomation), tissue sections were rewashed in buffer. Doublestain block (DakoCytomation) was applied for $3 \mathrm{~min}$, and the sections were incubated with the second antibody, CD20 (1:400, RT for 30 min; DakoCytomation) or CD3 (1:100, RT for $60 \mathrm{~min}$; Biocare Medical) and then rewashed. Fast-Red substrate (Biocare Medical) was used as the chromogen for the second antibody. Tissue sections were then counterstained with hematoxylin.

\section{Western Blot Analysis of HDM4 and p21 in Mantle Cell Lymphoma Cell Lines and Tumors}

To confirm expression of HDM4, cell extracts were prepared $^{27}$ from all four mantle cell lymphoma cell lines, and from peripheral blood of patients with leukemic-phase mantle cell lymphoma. Proteins were resolved on $12 \%$ sodium dodecyl sulfatepolyacrylamide gels. After electrophoresis, proteins were electrotransferred to nitrocellulose filters (Hybond-ECL; Amersham Corporation, Arlington Heights, IL, USA), probed with antibodies specific for HDM4 (1:1000), HDM4-S (1:250), and p21 (1:100), respectively, and detected by chemiluminescence (Amersham Biosciences, Little Chalfont, Buckinghamshire, UK). After initial probing, blots were reprobed with an antibody specific for actin (1:1000, mouse monoclonal; Sigma Aldrich, St Louis, MO, USA) to assess protein loading.

\section{Quantitative Real-Time-PCR and Direct Sequencing of PCR Products}

To characterize the splicing variants of HDM4, quantitative real-time polymerase chain reaction (RT-PCR) assays were performed as described by Bartel et $a l^{6}$ and calculated with the $2-\Delta \Delta$ Ct method using $\beta$-actin as endogenous control. Peripheral blood specimens obtained from untreated mantle cell lymphoma patients were used for analysis of HDM4. Commercially available CD19 + B-lymphocyte cDNAs were used as a control. Briefly, total RNA was extracted from CD19+ B-cells isolated from $2 \mathrm{ml}$ of EDTA-anticoagulated peripheral blood using the magnetic cell-sorting method (Miltenyi Biotec, Auburn, CA, USA). After treatment with RNase-free DNase, total RNA was used for complementary DNA synthesis using an oligo-dT primer. HDM4 and HDM4-S were amplified using the forward primer $5^{\prime}$-CAAGCCCTCTCTATGATATGCT-3' and reverse primer 5'-GCTCTGAGGTAGGCAGTGT G-3'. The PCR consisted of 35 cycles with $30 \mathrm{~s}$ of denaturation at $95^{\circ} \mathrm{C}, 30 \mathrm{~s}$ of primer annealing at $58^{\circ} \mathrm{C}$, and $30 \mathrm{~s}$ of synthesis at $72^{\circ} \mathrm{C}$. Amplified products were run on $8 \%$ polyacrylamide gels and detected by ethidium bromide stain. The amplified bands were excised, extracted, reamplified, and submitted for direct sequence analysis. Sequencing was performed using ABI Big Dye terminator cycle sequencing chemistry (Applied Biosystems, Foster City, CA).

\section{p53 Gene Mutation Analysis}

We performed p53 gene mutational analysis using specimens from three patients with leukemic-phase mantle cell lymphoma and two mantle cell lymphoma cell lines. Genomic DNA was extracted from $2 \mathrm{ml}$ of EDTA-anticoagulated peripheral blood using standard methods and analyzed for p53 mutations using the commercially available VariantSEQr kit (Applied Biosystems). Briefly, 12.5 ng of genomic DNA was used for each PCR. A total of 17 primer pairs were used for amplification of both exons and introns of p53 genomic DNA. The fluorescently labeled amplified products were analyzed using universal sequencing primers, BigDye Terminator v3.0 chemistry, and the Applied Biosystems 3730 Genetic Analyzer. These analyses cover more than $99 \%$ of the $p 53$ gene mutations reported in the website: http://www.ensembl.org/Homo_sapiens/ geneview ?gene $=$ ENSG00000141510\&db = \&_gene_ sequence $=1$.

\section{Inhibition of HDM4 Expression by siRNA}

Small interfering (si) RNA targeted against exons 3 and 4 of the HDM4 gene was used (Ambion Inc., Austin, TX, USA). Transient transfection of Mino and SP53 cells with siRNA was performed through electroporation using an electroporator (Amaxa Biosystems, Gaithersburg, MD, USA). Approximately $2 \times 10^{6}$ cells were transfected with $8 \mu \mathrm{M}$ of HDM4 siRNA using the Nucleofector kit T (Mino) or $\mathrm{V}$ (SP53) (Amaxa Biosystems) according to the manufacturer's protocol. Cells were collected at $20 \mathrm{~h}$ after transfection. Total RNA was extracted for quantitative RT-PCR analysis to determine the mRNA levels of full length HDM4 (HDM4-FL) and its splicing variants. Whole-cell protein lysates were prepared for western blot analysis using anti-HDM4 and anti-p21 antibodies. Cell viability was evaluated by using the Vi-CELL Series Cell Viability Analyzer (Beckman Coulter, Miami, FL, USA).

\section{Analysis of Cell Proliferation and Apoptosis}

After HDM4 inhibition by siRNA in Mino and SP53 cells, proliferation and apoptosis were assessed 
by double immunocytochemical staining using antibodies specific for Ki-67 (nuclear, brown) and caspase-3 (cytoplasmic, red) (Ki-67 plus caspase-3 prediluted double stain antibody, Biocare Medical). This staining was performed on cytospin-prepared slides of mantle cell lymphoma cell lines at different time points after transfection. The cell block slides were incubated with primary antibody at room temperature for $60 \mathrm{~min}$, followed by incubation at room temperature for 30 min using the Double Stain kit \#2 (Biocare Medical). Tissue sections were rinsed and counterstained with hematoxylin.

\section{Statistical Analyses}

Differences between groups were compared statistically using the Student's $t$-test or the Fisher's exact test. $P$ values less than 0.05 were deemed as statistically significant.

\section{Results}

\section{Clinicopathologic Characteristics}

A total of 25 mantle cell lymphoma patients were studied. There were 16 men and 9 women, with a median age of 61 years (range 30-87) and a median survival of 34 months. Six of these patients presented with leukemic phase of mantle cell lymphoma, five men and one woman, with a median age of 63 years (range 53-68) and a median survival of 7 months.

\section{Frequency of HDM4 Expression in Reactive Lymph Node and Mantle Cell Lymphoma}

In reactive lymph nodes $(n=19)$, germinal center cells expressed HDM4 in both the nucleus and cytoplasm. By contrast, mantle zone B-cells only focally and dimly expressed HDM4 in the nucleus and cytoplasm of 6 of 19 cases (Figure 1a; Table 1).
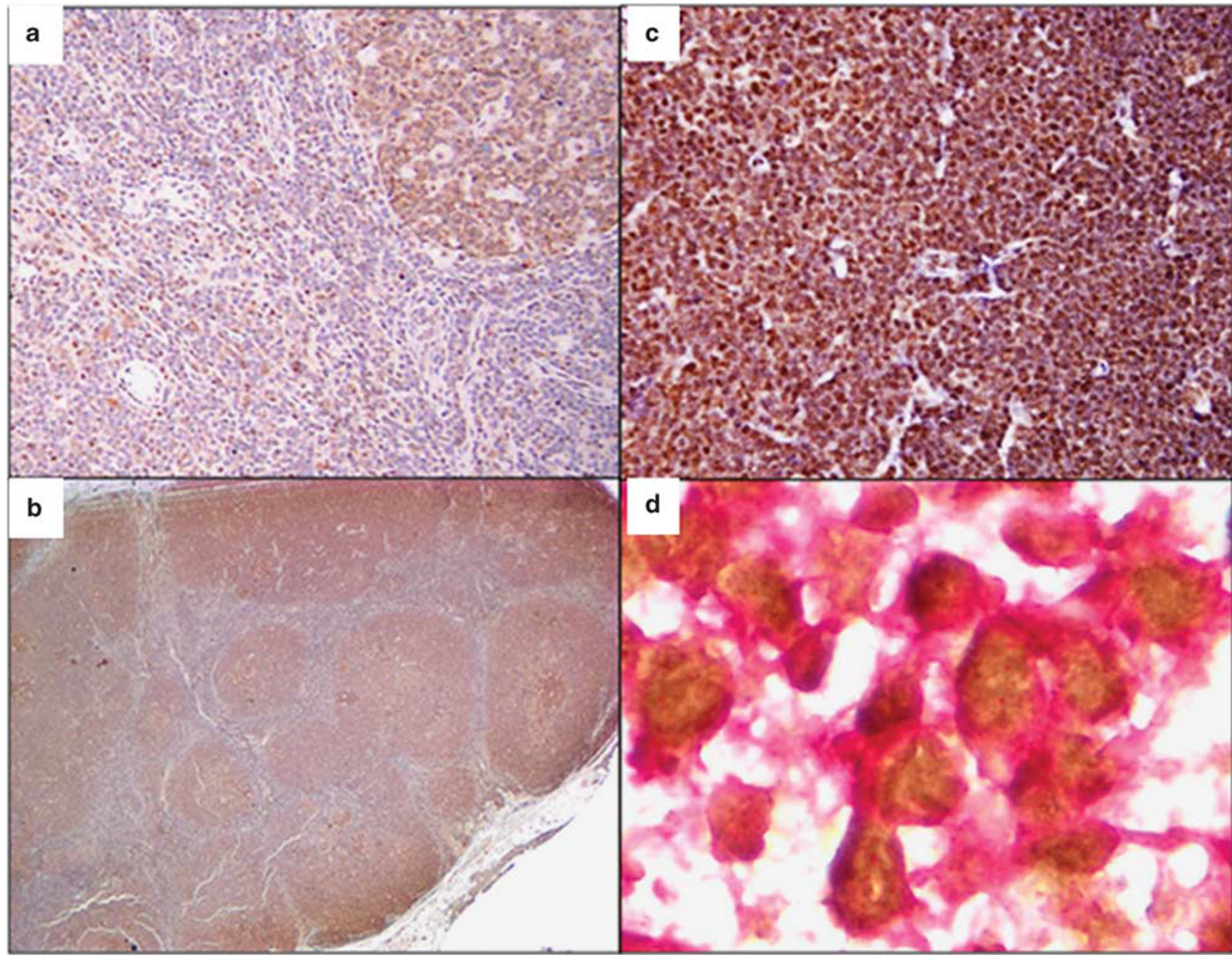

Figure 1 Immunohistochemical analysis of HDM4 in mantle cell lymphoma involving lymph nodes. (a) reactive, $\times 200$; (b) mantle cell lymphoma, $\times 20$; (c) mantle cell lymphoma, $\times$ 400; (d) mantle cell lymphoma, double staining with CD20 (red, membranous) and HDM4 (brown, nuclear), $\times 1000$ 
In mantle cell lymphoma tumors, HDM4 was significantly $(P<0.001)$ expressed diffusely in the cell nuclei in 18 of 19 (95\%) cases (Figures $1 \mathrm{~b}$ and c). In one case of mantle cell lymphoma with a focal mantle zone pattern, HDM4 was expressed more brightly in benign germinal center B-cells than in the neoplastic cells. Double staining with CD20/ HDM4 or CD3/HDM4 of mantle cell lymphoma tumors showed nuclear expression of HDM4 and cytoplasmic and membranous expression of CD20 (Figure 1d).

For comparison, HDM4 expression was assessed in other types of B-cell non-Hodgkin lymphoma (Table 1; Figures 2a-d). HDM4 was expressed in 8 of $11(72 \%)$ chronic lymphocytic leukemia/small lymphocytic lymphoma, 13 of 16 (94\%) follicular lymphoma (all 6 grade 1, 6 of 7 grade 2, and 1 of 3 grade 3), 16 of $20(80 \%)$ diffuse large B-cell lymphoma, and 5 of 6 (83\%) extranodal marginal zone B-cell lymphoma of mucosa-associated lymphoid tissue.

Immunohistochemistry (Figure 2) and western blot analysis of p21 in B-cell tumors expressing high levels of HDM4 revealed variable p21 protein levels. Endogenous p21 level was lower in mantle cell lymphoma than in other types of B-cell nonHodgkin lymphoma. Three of four mantle cell lymphoma cell lines (SP53, Granta 519, and Mino) and two patients with leukemic phase of mantle cell lymphoma showed decreased p21 levels compared to normal CD19-positive B-cells. Interestingly, the Z-138 mantle cell lymphoma cell line, derived from a case of mantle cell lymphoma in blastoid transformation, expressed high levels of p21 (Figure 2f).

Western blot analysis confirmed expression of HDM4-S and HDM4-FL proteins in mantle cell lymphoma tumors and cell lines (Figure 3a).

\section{HDM4 Transcripts are Aberrantly Overexpressed in Mantle Cell Lymphoma}

RT-PCR performed on six leukemic-phase mantle cell lymphoma specimens revealed the presence of two splicing forms of HDM4 mRNA. The RT-PCR reaction products were sequenced and the presence of a HDM4 variant with a short internal deletion of 68 base pairs (bp) was confirmed as shown earlier. ${ }^{2,24}$ The expression of both splicing variants, HDM4-S (containing only the p53-binding domain) and HDM4-FL, was increased compared with that of normal CD19+ B-cells. The HDM4-S to HDM4-FL ratio was significantly increased compared with that of normal CD19 + B-cells $(P<0.02)$ (Figure 3b). The HDM4-S to HDM4-FL ratio in the four mantle cell lymphoma cell lines was different. This ratio was increased in Z-138 and Mino, but not increased in SP53 and Granta 519 (Figure 3b). The HDM4-FL transcript was predominant in all normal tissues analyzed. As a control, we analyzed three non- mantle cell lymphoma cell lines, OCI-LY3 and LR (diffuse large B-cell lymphoma of the activated Bcell type), and DOHH2 (derived from a patient with follicular lymphoma). We found that the HDM4-S to HDM4-FL ratio was $0.11,0.46$, and 0.24 in OCI-LY3, $\mathrm{LR}$, and DOHH2, respectively (data not shown).

\section{Cell Growth and Apoptosis in Mantle Cell Lymphoma Cell Lines after Inhibition of HDM4 by Using siRNA}

After introducing siRNA to inhibit HDM4 in the SP53 and Mino cell lines (both carry mutant p53), p21 levels were increased (Figure 4). Immunocytochemical analysis showed increased p21 and active caspase-3-positive cells. p21 positivity was observed in $10.5 \pm 6.4 \%$ cells (control siRNA) versus $38.5 \pm 4.9 \%$ cells (HDM4-specific siRNA) in SP53 $(P=0.011)$, and $3.5 \pm 2.1 \%$ cells (control siRNA) versus $8.0 \pm 5.7 \%$ cells (HDM4-specific siRNA) in Mino (Figure 4). p21 expression was observed mostly in the cytoplasm of cells treated with the siRNA control, whereas it was predominantly nuclear in cells treated by HDM4-specific siRNA. Active caspase- 3 activity was observed in $6.0 \pm 4.2 \%$ cells (control siRNA) versus $21.0 \pm 8.5 \%$ cells (HDM4-specific siRNA), and $5.0 \pm 2.8 \%$ cells (siRNA control) versus $8.5 \pm 2.1 \%$ cells (HDM4-specific siRNA) $(P=0.045)$ in the SP53 and Mino cell lines, respectively. Proliferation rate assessed by Ki-67 positivity was observed in $67.0 \pm 25.4 \%$ cells (control siRNA) versus $84.0 \pm 14.1 \%$ cells (HDM4-specific siRNA) in the SP53 cell line, and $70.0 \pm 11.3 \%$ cells (control siRNA) versus $71.5 \pm 4.9 \%$ cells (HDM4-specific siRNA) in Mino (Figure 4; Table 2).

\section{p53 Gene Mutation Analysis in Mantle Cell Lymphoma Tumors and Cell Lines}

All leukemic-phase mantle cell lymphoma tumors analyzed showed the same mutations: one missense mutation in exon 4, base change $119 \mathrm{C}>\mathrm{G}$, amino acid change P267R; and two non-coding mutations in introns 8 and 9, respectively (Table 3). All three mutations were also identified in both mantle cell lymphoma cell lines tested: Mino and SP53. In addition, Mino had a missense mutation in exon 5, base change $65 \mathrm{~T}>\mathrm{G}$, amino acid change V342G, and SP53 had a missense mutation in exon 11, base change $567 \mathrm{G}>\mathrm{R}$, amino acid change $\mathrm{G} 784[\mathrm{~S}, \mathrm{G}]$.

\section{Discussion}

Alterations of the p53-HDM2/HDM4 pathway have been shown to have an important function in the pathogenesis of a variety of tumors. ${ }^{5,6}$ However, the role of this pathway in mantle cell lymphoma is not well explored. Our results show that HDM4 expression is frequently upregulated in mantle cell lymphoma compared with mantle zone B-cells in 
reactive lymph nodes. This study provides evidence that HDM4 protein is upregulated at the transition from normal mantle zone to germinal center B-cells, and that this pattern in preserved in B-cell tumors. Given the fact that mantle zone B-cells are considered to be in the resting state or in the G0 phase of

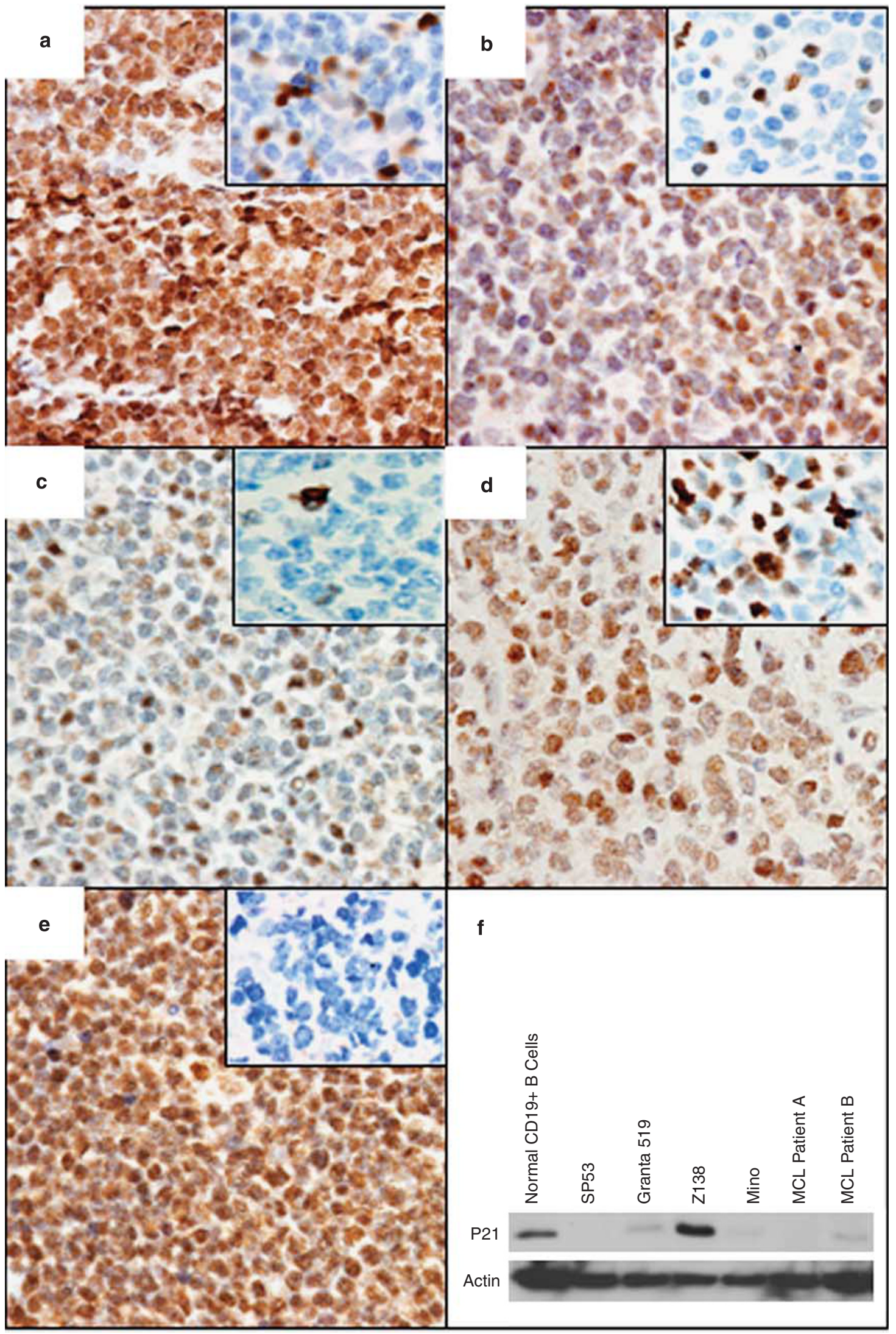


the cell cycle ${ }^{28}$ this raises the possibility that HDM4 is a marker for lymphocytes entering into the cell cycle.

Several splicing variants of HDM4 have been described including HDM4-A, HDM4-G, HDM4-S (human homolog of MDMX-S in mouse), and HDMX211 (isolated from thyroid tumor cell line, ARO). ${ }^{29}$ HDM-A lacks exon 9 sequences encoding the acidic domain and, as a result, cannot stabilize HDM2 and is susceptible to HDM2-initiated degradation. ${ }^{13}$ HDM4-G lacks exons 3-6 and cannot bind to p53. ${ }^{13}$ HDM4-S is a truncated protein containing only the p53-binding domain and is much more
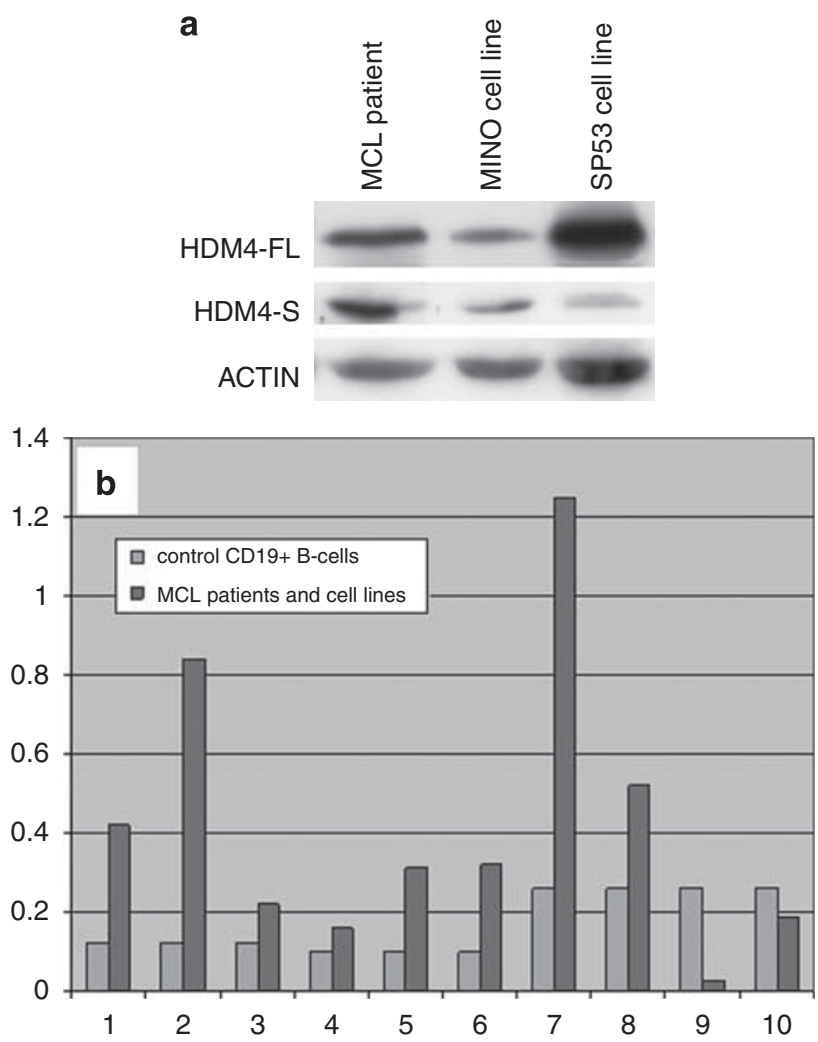

Figure 3 (a) Western blot analysis showing expression of HDM4-S and HDM4-FL in mantle cell lymphoma patients and cell lines; (b) Quantitative RT-PCR analysis showing HDM4-S to HDM4-FL ratio in mantle cell lymphoma tumors and cell lines. Series 1: control CD19 + B-cells; series 2: Mantle cell lymphoma patients and cell lines; 1-6: Mantle cell lymphoma patients; 7: Z138; 8: Mino; 9: SP53; 10: Granta. $(P=0.02)$. effective than HDM4-FL in inhibiting p53-mediated transcriptional activation and induction of apoptosis. It is generated by the loss of exon 6 , which produces a shift of the reading frame, resulting in a premature stop codon and an internal deletion of $68 \mathrm{bp} .{ }^{30}$ It has been shown that the HDM4-S mRNA splice variant is overexpressed and is associated with poor prognosis in soft tissue sarcoma. ${ }^{6}$ HDM4$S$ binds tightly to p53 and inhibits p53-mediated transcriptional activation inducing apoptosis. It also has been suggested that the ratio of HDM4-S to HDM4-FL may be more important than their absolute expression levels because both proteins compete for p53 binding. It also has been shown that HDMX-S has a greater affinity for p53 compared with HDM4-FL. ${ }^{30}$

We observed that both HDM4-S and HDM4-FL are increased in mantle cell lymphoma cells compared with normal CD19 + B-cells. In addition, there was an increased HDM4-S to HDM4-FL mRNA ratio in mantle cell lymphoma cells compared with reactive B-cells. Interestingly, the HDM4-S to HDM4-FL ratio differed in the mantle cell lymphoma cell lines tested. There was a substantially increased HDM4-S to HDM4-FL ratio in Z-138 and Mino. The ratio of HDM4-S to HDM4-FL was not increased in SP-53 or Granta 519 (cell lines with p53 loss of heterozygosity and loss of p16). The mechanisms involved in the elevated transcript levels of the HDM4 gene are unknown. A detailed analysis of the Granta 519 and Z-138 cell lines revealed complex karyotypes but no abnormalities at 1q32 where the HDM4 gene is located..$^{31}$ On the basis of sequencing data limited to the hot spots, SP53 has been considered as a mantle cell lymphoma cell line with wild-type p53. ${ }^{25}$

To confirm the p53 mutational status, we used 17 pairs of primers to amplify both exons and introns of p53 genomic DNA that are reported to cover more than $99 \%$ of the known p53 gene mutations. We have found that both Mino and SP53 share the same three mutations as do three of the leukemic-phase mantle cell lymphoma tumors analyzed. Mino was developed from the peripheral blood of a 64-yearold man during leukemic transformation of mantle cell lymphoma, ${ }^{32}$ whereas SP-53 was established from the peripheral blood of a 58-year-old woman with leukemic transformation of an intermediate lymphocytic lymphoma, an older term for mantle cell lymphoma. ${ }^{33}$ The fact that the same $p 53$

Figure 2 Immunohistochemical analysis of HDM4 and p21 (insets) in different types of B-cell lymphoma. (a) Follicular lymphoma involving the lymph node. There is extensive nuclear and cytoplasmic HDM4 expression and p21 is expressed in the nuclei of a subset of tumor cells (inset); (b) marginal zone B-cell lymphoma. HDM4 is expressed in a weak nuclear and cytoplasmic staining pattern. Scattered p21-positive cells are present (inset); (c) chronic lymphocytic leukemia/small lymphocytic lymphoma. HDM4 is expressed in a subset of the tumor cells with very few p21-positive cells (inset); (d) diffuse large B-cell lymphoma of germinal center origin. Most tumor cells are HDM4 positive with strong nuclear p21 expression (inset); (e) mantle cell lymphoma. The neoplastic cells are strongly positive for HDM4 and negative for p21 (inset). Original magnification for all images $\times 400$. (f) Western blot analysis showed that endogenous p21 level is higher in normal CD19+ B-cells than in patients with mantle cell lymphoma and most mantle cell lymphoma cell lines. 


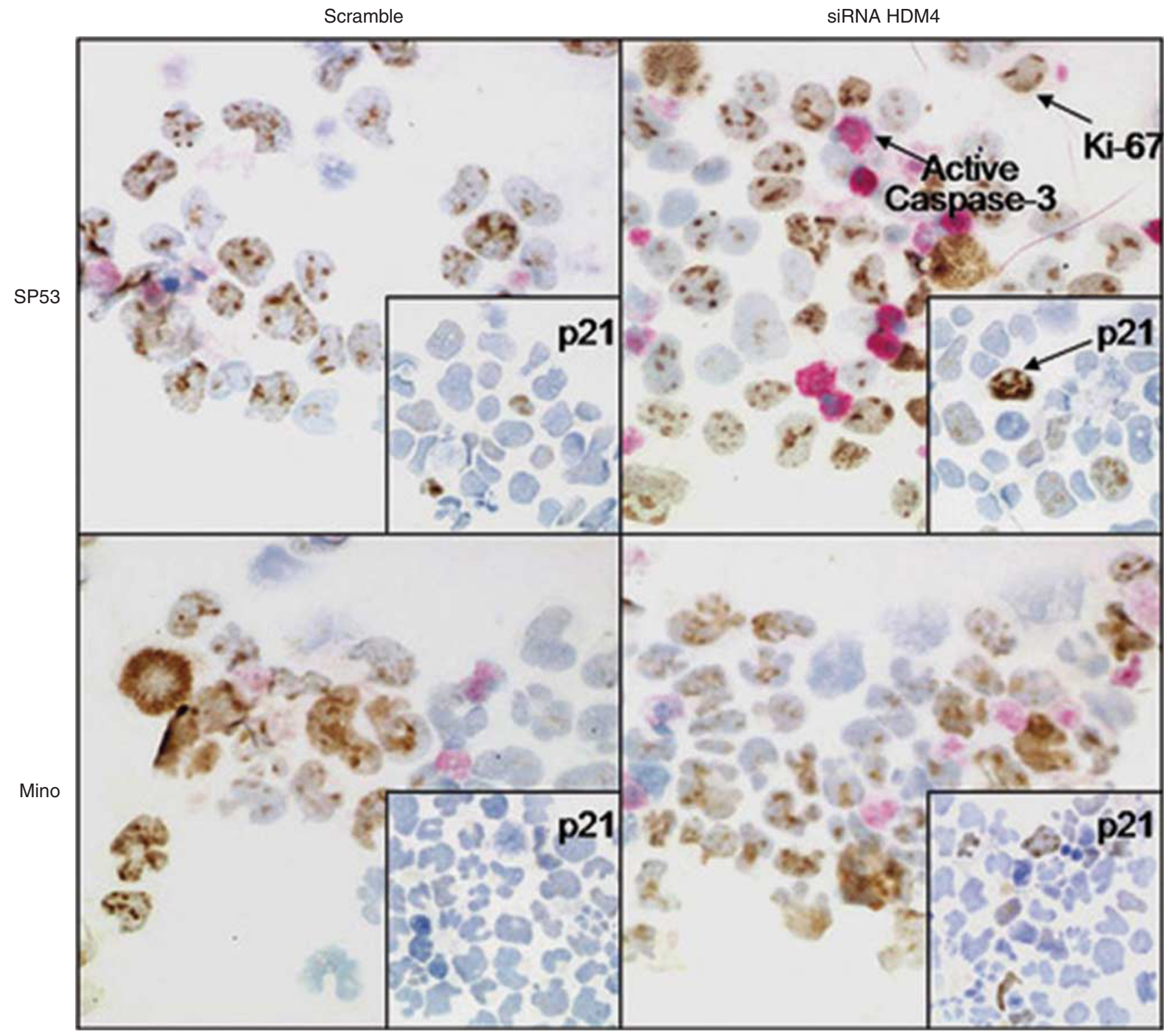

Figure 4 Inhibition of HDM4 by small interfering (si) RNA induces active caspase-3 and p21 expression in mantle cell lymphoma cell lines. Double immunostaining reveals upregulation of active caspase 3 (red) without significant increase in nuclear Ki-67 staining (brown) in SP53 and Mino after inhibition of HDM4 using siRNA compared with control (scramble siRNA). Similar upmodulation of p21 (insets) was found in SP53 and Mino cells treated with HDM4-specific siRNA.

Table 2 Increased cell growth and apoptotic events after introducing siRNA inhibition of HDM4 in MCL cell lines

\begin{tabular}{|c|c|c|c|c|c|c|}
\hline & \multicolumn{2}{|c|}{$p 21$} & \multicolumn{2}{|c|}{ Active caspase-3 } & \multicolumn{2}{|c|}{ Ki-67 } \\
\hline & $\begin{array}{l}\text { siRNA } \\
\text { control }\end{array}$ & $\begin{array}{l}\text { SiRNA } \\
\text { HDM4 }\end{array}$ & $\begin{array}{l}\text { siRNA } \\
\text { control }\end{array}$ & $\begin{array}{l}\text { siRNA } \\
\text { HDM4 }\end{array}$ & $\begin{array}{l}\text { siRNA } \\
\text { control }\end{array}$ & $\begin{array}{l}\text { siRNA } \\
\text { HDM4 }\end{array}$ \\
\hline Mino (\%) & $3.5 \pm 2.1$ & $8.0 \pm 5.7$ & $5.0 \pm 2.8$ & $8.5 \pm 2.1^{*}$ & $70.0 \pm 11.3$ & $71.5 \pm 4.9$ \\
\hline SP53 (\%) & $10.5 \pm 6.4$ & $38.5 \pm 4.9^{*}$ & $6.0 \pm 4.2$ & $21.0 \pm 8.5$ & $67.0 \pm 25.4$ & $84.0 \pm 14.1$ \\
\hline
\end{tabular}

${ }^{*} P<0.05$.

mutations are present in mantle cell lymphoma patient tumors and cell lines suggests that the mutations observed in the cell lines are not acquired during in vitro culture. Thus, Mino and SP53 are representative of mantle cell lymphoma and can be used as valuable and convenient tools to study the disease pathogenesis. SP53 should no longer be considered as being wild type for $p 53$. 
Table 3 Mutation analysis of p53 gene status in three MCL patients ${ }^{\mathrm{a}}$ and two MCL cell lines

\begin{tabular}{|c|c|c|c|c|c|c|c|}
\hline & Base_change & Site & Position & Type & Effect & AA_Change & $3 D$ location \\
\hline 1 & $119 \mathrm{C}>\mathrm{G}$ & Exon 4 & 119 & Sub & Missense & P267R & DNA core-binding domain, L1 loop \\
\hline 2 & $38 \mathrm{C}>\mathrm{G}$ & Intron 8 & 38 & Sub & Non-coding & - & $\mathrm{N}$-terminal; transactivation \\
\hline 3 & 41-56 Del & Intron 9 & 41 & Del & Non-coding & - & $\mathrm{N}$-terminal; transactivation \\
\hline Mino & $119 \mathrm{C}>\mathrm{G}$ & Exon 4 & 119 & Sub & Missense & P267R & DNA core-binding domain, L1 loop \\
\hline Mino & $65 \mathrm{~T}>\mathrm{G}$ & Exon 5 & 65 & Sub & Missense & V342G & N-terminal; transactivation \\
\hline Mino & $38 \mathrm{C}>\mathrm{G}$ & Intron 8 & 38 & Sub & Non-coding & - & $\mathrm{N}$-terminal; transactivation \\
\hline Mino & 41-56 Del & Intron 9 & 41 & Del & Non-coding & - & N-terminal; transactivation \\
\hline SP53 & $119 \mathrm{C}>\mathrm{G}$ & Exon 4 & 119 & Sub & Missense & P267R & DNA core-binding domain, L1 loop \\
\hline SP53 & $38 \mathrm{C}>\mathrm{G}$ & Intron 8 & 38 & Sub & Non-coding & - & $\mathrm{N}$-terminal; transactivation \\
\hline SP53 & 41-56 Del & Intron 9 & 41 & Del & Non-coding & - & N-terminal; transactivation \\
\hline SP53 & $567 \mathrm{G}>\mathrm{R}$ & Exon 11 & 567 & Sub & Missense & $\mathrm{G} 784[\mathrm{~S}, \mathrm{G}]$ & C-terminal; tetramerization \\
\hline
\end{tabular}

${ }^{a}$ All three MCL patients have the same three mutations listed as 1-3.

AA: amino acid; Del: deletion; P267R: proline at position 267 replaced by arginine; sub: substitution; V342G: valine at position 342 replaced by glycine; G784[S,G]: heterozygous mutation, glycine at position 784 replaced by serine.

Three of six leukemic-phase mantle cell lymphoma tumors assessed had p53 mutations. All three tumors showed identical p53 mutations. One of the mutations is present in exon 4 , with base change $119 \mathrm{C}>\mathrm{G}$, and amino acid change P267R. This mutation has also been found in five patients with B-lymphoblastic leukemia/lymphoma in our previous studies (four have 119C $>\mathrm{G}$, one has $119 \mathrm{C}>\mathrm{S}){ }^{24}$ It is located in the Loop-L1 of the central core domain, which contains the sequencespecific DNA-binding activity of the p53 protein (residues 102-292). ${ }^{34}$ It is been reported that most p53 mutations occur in this region and result in loss of DNA binding. A recent multi-institutional study has reported that mutations in p53 DNA-binding domains are the strongest predictors of poor overall survival in patients with diffuse large B-cell lymphoma. ${ }^{35}$ The p53 mutations identified in our studies are not reported in their study.

In the literature, overexpression of HDM4 seems to be associated with wild-type $p 53$. Riemenschneider et $a l^{4}$ reported that amplification and overexpression of HDM4 was exclusively found in glioblastomas with wild-type $p 53$, more specifically in tumors without hot spot mutations, and they suggested that HDM4 amplification and overexpression represents a novel mechanism by which a small percentage of malignant gliomas might escape p53dependent growth control. Similar findings were shown in melanoma and ovarian cancer cell lines, ${ }^{7}$ as well as soft tissue sarcomas. ${ }^{6}$ However, we were unable to confirm an association between HDM4 overexpression and the presence of a wild-type p53 gene in any of the mantle cell lymphoma cases in this study. Unlike other studies, we sequenced the entire protein-coding portions of the gene. Of special interest was the high rate of mutations in exon 4 of the p53 gene, suggesting that it may be a mutation-prone region, in agreement with the findings of other reports. ${ }^{35}$

In addition, HDM4-S seems to be more effective than HDM4-FL at inhibiting p53-mediated transcriptional activation. Riemenschneider et $a l^{4}$ report that the ratio of HDM4-S to HDM4-FL is higher in glioblastomas than in gliomas, and Rallapalli et $a l^{30}$ describe that HDM4-S is upregulated in transformed cells and downregulated in low passage cells. These findings are consistent with the results of our studies on six leukemic-phase mantle cell lymphoma patient tumors, all of which were the blastoid variant, and all with an increased HDM4-S to HDM4-FL ratio. Interestingly, the cell line Z-138 that was derived from a patient with blastoid transformation of mantle cell lymphoma in the terminal phase of the disease exhibited the highest HDM4-S to HDM4-FL ratio and increased p21 levels. It is possible that because the HDM4-S variant comprises only the p53-binding domain and a few alternative C-terminal amino acids it can no longer interact with p21 and is, therefore, protected from HDM4-mediated degradation.

Larger scale studies of the p53 mutation status in mantle cell lymphoma patients will help validate a possible association between wild-type p53 and HDM4 overexpression or an increased HDM4-S to HDM4-FL ratio in mantle cell lymphoma. Given the fact that p53 mutations are uncommon in classic mantle cell lymphoma with a low proliferative activity, but are found in up to $30 \%$ of blastoid mantle cell lymphoma cases that have a high proliferation rate and are associated with poor prognosis, ${ }^{21,22}$ it might be true that most mantle cell lymphoma in leukemic phase have mutated p53. This is consistent with conventional cytogenetic data of mantle cell lymphoma cases that have shown chromosome 17 abnormalities more commonly in leukemic-phase mantle cell lymphoma. ${ }^{19}$

HDM4 inhibits p53-mediated transcriptional activation of several downstream targets, such as p21. Recent studies by Jin et $a l^{16}$ showed that HDM4 also promotes p53 and p21 degradation synergistically with HDM2. They also showed that MDMX (mouse homolog of HDM4) directly bound to p21 and mediated its proteasomal degradation, both 
independently and synergistically with MDM2. ${ }^{16}$ Depletion of MDMX leads to increased levels of endogenous p21 and results in G1 arrest. Knockdown of p21 by siRNA rescues G1 arrest by depletion of MDMX and MDM2 in p53-null cells. ${ }^{16}$ This is in accord with our observations of increased p21 protein expression after siRNA inhibition of HDM4. Immunocytochemical analysis also showed increased p21 and active caspase-3-positive cells, but no significant changes of Ki-67 expression, indicating increased apoptotic events. This implies a more direct and crucial role of HDM4 in regulating the cell cycle through cyclin-dependent kinase inhibitors and monitoring the p21 levels.

In summary, our data indicate that HDM4 overexpression is common in mantle cell lymphoma as well as in other types of B-cell NHL. Overexpression of the HDM4-S and HDM4-FL transcripts are observed in mantle cell lymphoma tumors and cell lines. Ablation of HDM4 induces p21 overexpression and leads to increased active caspase-3 but has no significant effect on proliferation. Our data also suggest that HDM4 may have a pathogenetic role in mantle cell lymphoma and be a promising therapeutic target in patients with this disease. In fact, there is growing interest in targeting the p53HDM2-HDM4 pathway as a new cancer therapy. Nutlin-3, an antagonist of p53-HDM2 interaction, has been shown to improve tumor cell response to chemotherapy in chronic lymphocytic leukemia ${ }^{36}$ and induce cell-cycle arrest and apoptosis in mantle cell lymphoma cell lines. ${ }^{37}$ Our study suggests that additional inhibition of HDM4 pathway may be important as part of a therapeutic regimen targeting p53 activation and controlling cell proliferation in patients with mantle cell lymphoma.

\section{Acknowledgements}

Part of this work was presented at the United States and Canadian Academy of Pathology Annual Meeting, Boston, MA, USA, March 7-13, 2009. We are grateful to Geneva Williams and LaKisha Rodgers for their assistance in formatting the figures and the manuscript.

\section{Disclosure/conflict of interest}

The authors declare no conflict of interest.

\section{References}

1 Toledo F, Wahl GM. Regulating the p53 pathway: in vitro hypotheses, in vivo veritas. Nat Rev Cancer 2006;6:909-923.

2 Shvarts A, Bazuine M, Dekker P, et al. Isolation and identification of the human homolog of a new p53binding protein, Mdmx. Genomics 1997;43:34-42.
3 Shvarts A, Steegenga WT, Riteco N, et al. MDMX: a novel p53-binding protein with some functional properties of MDM2. EMBO J 1996;15:5349-5357.

4 Riemenschneider MJ, Buschges R, Wolter M, et al. Amplification and overexpression of the MDM4 (MDMX) gene from 1q32 in a subset of malignant gliomas without TP53 mutation or MDM2 amplification. Cancer Res 1999;59:6091-6096.

5 Danovi D, Meulmeester E, Pasini D, et al. Amplification of Mdmx (or Mdm4) directly contributes to tumor formation by inhibiting p53 tumor suppressor activity. Mol Cell Biol 2004;24:5835-5843.

6 Bartel F, Schulz J, Bohnke A, et al. Significance of HDMX-S (or MDM4) mRNA splice variant overexpression and HDMX gene amplification on primary soft tissue sarcoma prognosis. Int J Cancer 2005;117: 469-475.

7 Ramos YF, Stad R, Attema J, et al. Aberrant expression of HDMX proteins in tumor cells correlates with wild-type p53. Cancer Res 2001;61:1839-1842.

8 Stad R, Ramos YF, Little N, et al. Hdmx stabilizes Mdm2 and p53. J Biol Chem 2000;275:28039-28044.

9 Marine JC, Francoz S, Maetens M, et al. Keeping p53 in check: essential and synergistic functions of Mdm2 and Mdm4. Cell Death Differ 2006;13:927-934.

10 Marine JC, Jochemsen AG. Mdmx as an essential regulator of p53 activity. Biochem Biophys Res Commun 2005;331:750-760.

11 Stad R, Little NA, Xirodimas DP, et al. Mdmx stabilizes p53 and Mdm2 via two distinct mechanisms. EMBO Rep 2001;2:1029-1034.

12 Sharp DA, Kratowicz SA, Sank MJ, et al. Stabilization of the MDM2 oncoprotein by interaction with the structurally related MDMX protein. J Biol Chem 1999;274:38189-38196.

13 de Graaf P, Little NA, Ramos YF, et al. Hdmx protein stability is regulated by the ubiquitin ligase activity of Mdm2. J Biol Chem 2003;278:38315-38324.

14 Parant J, Chavez-Reyes A, Little NA, et al. Rescue of embryonic lethality in Mdm4-null mice by loss of Trp53 suggests a nonoverlapping pathway with MDM2 to regulate p53. Nat Genet 2001;29:92-95.

15 Linares LK, Hengstermann A, Ciechanover A, et al. HdmX stimulates Hdm2-mediated ubiquitination and degradation of p53. Proc Natl Acad Sci USA 2003;100:12009-12014.

16 Jin Y, Zeng SX, Sun XX, et al. MDMX promotes proteasomal turnover of p21 at G1 and early $S$ phases independently of, but in cooperation with, MDM2. Mol Cell Biol 2008;28:1218-1229.

17 Bodrug SE, Warner BJ, Bath ML, et al. Cyclin D1 transgene impedes lymphocyte maturation and collaborates in lymphomagenesis with the myc gene. EMBO J 1994;13:2124-2130.

18 Lovec H, Grzeschiczek A, Kowalski MB, et al. Cyclin D1/bcl-1 cooperates with myc genes in the generation of B-cell lymphoma in transgenic mice. EMBO J 1994;13:3487-3495.

19 Onciu M, Schlette E, Medeiros LJ, et al. Cytogenetic findings in mantle cell lymphoma cases with a high level of peripheral blood involvement have a distinct pattern of abnormalities. Am J Clin Pathol 2001;116:886-892.

20 Fernandez V, Hartmann E, Ott G, et al. Pathogenesis of mantle-cell lymphoma: all oncogenic roads lead to dysregulation of cell cycle and DNA damage response pathways. J Clin Oncol 2005;23:6364-6369. 
21 Greiner TC, Moynihan MJ, Chan WC, et al. p53 mutations in mantle cell lymphoma are associated with variant cytology and predict a poor prognosis. Blood 1996;87:4302-4310.

22 Louie DC, Offit K, Jaslow R, et al. p53 overexpression as a marker of poor prognosis in mantle cell lymphomas with $\mathrm{t}(11 ; 14)(\mathrm{q} 13 ; \mathrm{q} 32)$. Blood 1995;86: 2892-2899.

23 Kienle D, Katzenberger T, Ott G, et al. Quantitative gene expression deregulation in mantle-cell lymphoma: correlation with clinical and biologic factors. J Clin Oncol 2007;25:2770-2777.

24 Han X, Garcia-Manero G, McDonnell TJ, et al. HDM4 (HDMX) is widely expressed in adult pre-B acute lymphoblastic leukemia and is a potential therapeutic target. Mod Pathol 2007;20:54-62.

25 Amin HM, McDonnell TJ, Medeiros LJ, et al. Characterization of 4 mantle cell lymphoma cell lines. Arch Pathol Lab Med 2003;127:424-431.

26 Medeiros LJ, Estrov Z, Rassidakis GZ. Z-138 cell line was derived from a patient with blastoid variant mantle cell lymphoma. Leuk Res 2006;30:497-501.

27 Bueso-Ramos CE, Rocha FC, Shishodia S, et al. Expression of constitutively active nuclear-kappa B RelA transcription factor in blasts of acute myeloid leukemia. Hum Pathol 2004;35:246-253.

28 Shen Y, Iqbal J, Xiao L, et al. Distinct gene expression profiles in different B-cell compartments in human peripheral lymphoid organs. BMC Immunol 2004;5:20.

29 Giglio S, Mancini F, Gentiletti F, et al. Identification of an aberrantly spliced form of HDMX in human tumors: a new mechanism for HDM2 stabilization. Cancer Res 2005;65:9687-9694.
30 Rallapalli R, Strachan G, Cho B, et al. A novel MDMX transcript expressed in a variety of transformed cell lines encodes a truncated protein with potent p53 repressive activity. J Biol Chem 1999;274:8299-8308.

31 Mestre-Escorihuela C, Rubio-Moscardo F, Richter JA, et al. Homozygous deletions localize novel tumor suppressor genes in B-cell lymphomas. Blood 2007;109:271-280.

32 Lai R, McDonnell TJ, O’Connor SL, et al. Establishment and characterization of a new mantle cell lymphoma cell line, Mino. Leuk Res 2002;26: 849-855.

33 Daibata M, Kubonishi I, Eguchi T, et al. The establishment of Epstein-Barr virus nuclear antigen-positive (SP-50B) and Epstein-Barr virus nuclear antigennegative (SP-53) cell lines with $t(11 ; 14)(q 13 ; q 32)$ chromosome abnormality from an intermediate lymphocytic lymphoma. Cancer 1989;64:1248-1253.

34 Cho Y, Gorina S, Jeffrey PD, et al. Crystal structure of a p53 tumor suppressor-DNA complex: understanding tumorigenic mutations. Science 1994;265:346-355.

35 Young $\mathrm{KH}$, Leroy $\mathrm{K}$, Moller $\mathrm{MB}$, et al. Structural profiles of TP53 gene mutations predict clinical outcome in diffuse large B-cell lymphoma: an international collaborative study. Blood 2008;112:3088-3098.

36 Coll-Mulet L, Iglesias-Serret D, Santidrian AF, et al. MDM2 antagonists activate p53 and synergize with genotoxic drugs in B-cell chronic lymphocytic leukemia cells. Blood 2006;107:4109-4114.

37 Drakos E, Atsaves V, Li J, et al. Stabilization and activation of p53 downregulates mTOR signaling through AMPK in mantle cell lymphoma. Leukemia 2009;23:784-790. 